\title{
Ultra-Fast Outflows in Typical Redshift 2 Star-Forming Galaxies
}

\author{
Orianne Roos ${ }^{1}$ and Frédéric Bournaud ${ }^{1}$ \\ ${ }^{1}$ CEA-Saclay, 91190 Gif-sur-Yvette, France \\ email: orianne.roos@cea.fr
}

\begin{abstract}
Galactic outflows are observed in star-forming galaxies up to high redshift. Still, their physical origins are not well understood, and most simulations fail to reproduce all observed parameters from first principles. With the POGO simulations (Physical Origins of Galactic Outflows), we model AGN and stellar feedback (FB) based on physical assumptions, and investigate their impact on the outflow parameters and on the host-galaxy at very high resolution $(1.5 \mathrm{pc})$. Here, we show that AGN and stellar FB couple non-linearly, and that the mass loading of the resulting outflow highly depends on the mass of the host.
\end{abstract}

Keywords. galaxies: high-redshift — galaxies: active — galaxies: outflows - stars: outflows

\section{Introduction}

Observations show ubiquitous galactic outflows in star-forming galaxies (SFGs) at all redshifts for which interstellar absorption features are accessible (Steidel et al. 2010). Such galactic outflows are mostly generated by internal feedback (FB): young stars and supernovae (SNe) are known to drive outflows with a fairly high mass loading, but limited velocity of $100-500 \mathrm{~km} / \mathrm{s}$ (e.g. Bournaud et al. 2014), while active galactic nuclei (AGNs) can trigger ultra-fast outflows (UFOs) up to $3000-30000 \mathrm{~km} / \mathrm{s}$ (Chartas et al. 2014), but with smaller gas densities and outflow rates (Gabor \& Bournaud 2014).

Current high-resolution galaxy simulations include AGN or stellar FB recipes that create galactic outflows, but most of them fail to reproduce all observed outflow parameters on physical bases. For instance, the velocity of simulated AGN-driven winds corresponds to observations but not the mass loading, and vice-versa for stars-driven winds. In this paper, we present the POGO simulations: Physical Origins of Galactic Outflows. With very high resolution simulations of isolated star-forming disk galaxies at redshift 2 , we model for the first time AGN and stellar winds from first principles. We run a suite of 15 simulations with three galaxy masses $\left(\mathrm{Mgas}_{\text {gas }}=1.5,4.9\right.$ and $\left.11.5 \times 10^{10} \mathrm{M}_{\odot}\right)$, three FB configurations (AGN FB, stellar FB, and both) and three spatial resolutions (1.5, 3 and $6 \mathrm{pc}$ ), to identify the main outflow driver as a function of stellar mass and the effects of such outflows on the host-galaxy. Here, we investigate the mass loading of the outflows.

\section{Feedback models}

AGN feedback. Our AGN FB model is twofold : we consider standard thermal energy injection, and photo-ionization. Thermal energy is injected according to matter accretion onto the black hole $(\mathrm{BH})$, and is modeled using the standard recipe of FB described by Booth \& Schaye (2009). The effect of AGN radiation will be computed using the post-processing method presented in Roos et al. (2015). We have shown that neither AGN-driven winds (Gabor \& Bournaud 2013, 2014), nor AGN-driven winds and AGN photo-ionization coupled (Roos et al. 2015) can quench star formation in typical redshift 


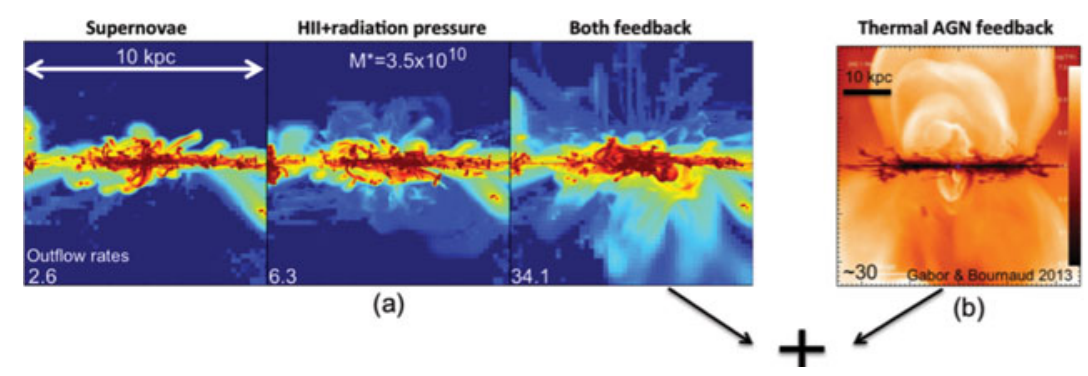

Figure 1. (a) Gas density of a $\mathrm{z} \sim 2$ SFG simulation run by F. Renaud. The galactic disk is shown edge-on. Dense star-forming regions are in red and diffuse outflowing gas is in light blue. The panels show outflows generated by SNe only (left), radiative FB from OB stars only (center), and both models combined (right). Outflow rates are indicated in $\mathrm{M}_{\odot} / \mathrm{yr}$ and clearly show the non-linearity of the coupling, but even with all stellar FB combined, it is not high enough to explain the missing baryons problem. (b) Comparison with thermal AGN FB in a similar SFG shown edge-on. Combining AGN and stellar FB at very high resolution may produce powerful outflows, with outflow rates compatible with the missing baryons problem.

2 SFGs, on a time-scale of a few hundreds of million years.

Stellar feedback. We combine three stellar FB models : kinetic and thermal energy injection from SNe (Dubois \& Teyssier 2008), and radiative pressure injection from young stars (Renaud et al. 2013). Figure 1 shows that injection of kinetic energy from SNe and radiative pressure from young stars couple non-linearly to produce strong winds (see also Hopkins et al. 2014). However, the resulting outflow rate is still too low to explain the observed IGM density (the "missing baryons problem", Sommer-Larsen 2006).

\section{Coupling of AGN and stellar feedback}

Similarly to the combination of several stellar FB models (Figure 1), AGN and stellar FB models could also couple non-linearly and produce very fast outflows, with important outflow rates. We launched our suite of simulations in order to verify this intuition, and now present our first conclusive and convincing results.

Low-mass $\mathrm{z} \sim \mathbf{2}$ SFG. For our low-mass SFG simulation $\left(\mathrm{Mgas}_{\mathrm{s}}=1.5 \times 10^{10} \mathrm{M}_{\odot}\right)$, we study two resolutions (1.5 and $3 \mathrm{pc}$ ), and three FB configurations : thermal AGN FB (hereafter, AGN); thermal and kinetic SN FB and radiative FB from young stars (hereafter, KThR); both (hereafter, KThR+AGN), which is the fiducial FB configuration. In the AGN configuration, residual thermal SN FB is maintained, in order to keep a realistic probability density function $(\mathrm{PDF})$ of the gas in the ISM. Accretion onto the $\mathrm{BH}$ is maintained in all runs: only AGN FB is shut down in the KThR configuration.

The outflow rate is the mass flux crossing the surface of a shell in a given time $d t$. The radii of the shells are in the range $14-50 \mathrm{kpc}$, and their center is that of the simulation box. To avoid the galactic disk, the portions of the shells which cross a $2.5 \mathrm{kpc}$ layer on each side of the galactic mid-plane are removed from the computation of the outflow rates. For each galaxy mass, the total SFR of the KThR configuration is comparable to that of the KThR+AGN configuration. In the AGN configuration, the SFR is higher since stellar FB has been minimized. This effect is artificial and is therefore not taken into account. The mass loading is defined as the ratio between the outflow rate of the simulation (KThR, AGN or KThR+AGN), and the SFR of the fiducial simulation.

AGN FB has been enabled after 115 Myrs of evolution. Figure 2 shows the evolution of the outflow rate as a function of time since then, for the three FB configurations 


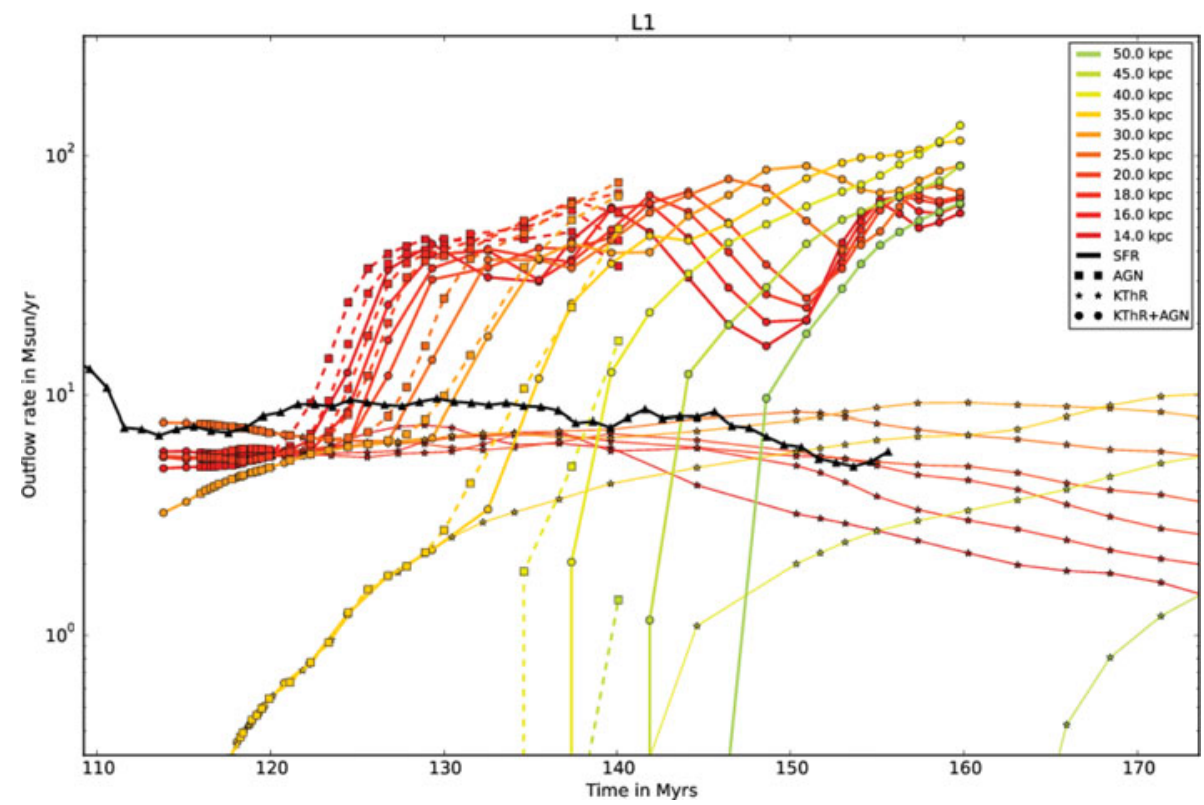

Figure 2. Outflow rate as a function of time for the low-mass SFG. The color gradient shows the radius of the shell through which the outflow rate is computed. SFR is shown in black. Circles correspond to KThR+AGN, stars to KThR and squares to AGN. AGN and stellar FB couple non-linearly: dense gas in the stellar outflow slows down the AGN outflow, resulting in an overall smaller mass loading compared to the AGN alone.

at low resolution $(3 \mathrm{pc})$. All three outflows have reached a steady state. While stellar outflows have a mass loading roughly equal to 1 , KThR + AGN outflows tend to a mass loading of $\sim 10$ and that of the AGN outflow is roughly double. This is likely due to the presence of dense gas in the outflow: it is brought there by the stellar component of the wind and decreases the overall velocity of the outflow. It remains to be examined whether the presence or absence of stellar FB modifies the $\mathrm{BH}$ accretion rate (BHAR) in a substantial way. The behaviour is similar at high resolution (1.5 pc), even if the BHAR is not converged.

For the AGN and KThR+AGN configurations, the outflowing gas is distributed in successive bursts, which have a temperature higher than the circumgalactic medium (CGM) (Figure 3, left). Therefore, assuming galactic outflows (even those mainly driven by the AGN) are smooth is not a good approximation. The right panel of Figure 3 shows that the velocity field of the outflowing gas is spherical at large distance in the gaseous halo. The outflowing gas reaches several $10^{3} \mathrm{~km} / \mathrm{s}$.

Intermediate-mass $\mathbf{z} \sim 2$ SFG. The mass of our intermediate-mass SFG is Mgas $=$ $4.9 \times 10^{10} \mathrm{M}_{\odot}$. As for the low-mass simulated SFG, we have a low-resolution run at $3 \mathrm{pc}$ and a high-resolution run at $1.5 \mathrm{pc}$. The same three FB configurations are used. AGN FB was enabled after 70 Myrs of evolution and all outflows have reached a steady state 20 Myrs after that. In contrast with the low-mass SFG, stellar winds are quite weak (mass loading of about 0.1). As for the low-mass SFG, both AGN and KThR+AGN outflows are powerful (mass loading $\sim 2$ ). This behaviour is in remarkable agreement with the observations of Bouché et al. (2010). Similarly to the low-mass SFG, AGN outflows grow faster than KThR+AGN outflows, but once the steady state is reached for both, they seem to have a comparable mass loading. Again, this is due to the presence of dense gas 

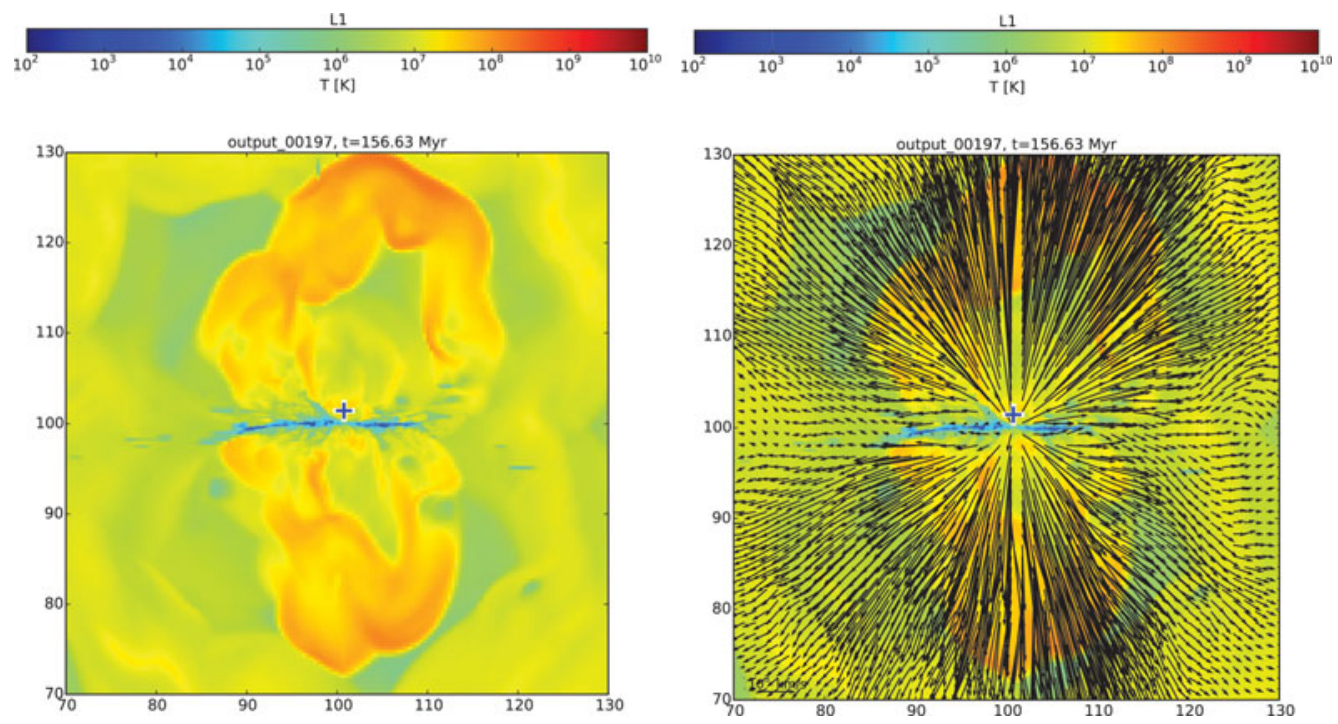

Figure 3. Left: Edge-on view of the low-mass KThR+AGN SFG simulation. The color gradient shows gas temperature. The axes are in kpc. The cross shows the location of the BH. AGN FB was enabled 40 Myrs ago. The outflowing gas is hotter than the CGM. Right: Same panel. The velocity field is super-imposed. The outflowing gas has a typical velocity of several $10^{3} \mathrm{~km} / \mathrm{s}$.

in the outflow, but as KThR outflows are weaker than for the low-mass SFG, the effect tends to disappear with time.

High-mass $\mathbf{z} \sim \mathbf{2}$ SFG. For the high-mass SFG $\left(\mathrm{M}_{\text {gas }}=11.5 \times 10^{10} \mathrm{M}_{\odot}\right)$, we only test KThR+AGN FB and run an extra simulation at $6 \mathrm{pc}$, in order to evolve the outflow until it reaches a stationary state. 15 Myrs after enabling AGN FB, the main driver of the outflow is the AGN and its mass loading is $\sim 2$.

\section{Acknowledgement}

We acknowledge support from the EC through grants ERC-StG-257720 and the CosmoComp ITN. Simulations were performed at TGCC and IDRIS under GENCI allocations 2013-GEN2192 and 2014-GEN2192. We also acknowledge PRACE for awarding us access to resource Curie, based in France at Bruyères-le-Châtel. The authors thank Florent Renaud for sharing the simulation outputs presented in Figure 1 (a).

\section{References}

Booth, C. M., \& Schaye, J. 2009, Mon. Not. R. Astron. Soc., 398, 53

Bouché, N., Dekel, A., Genzel, R., et al. 2010, Astrophys. J., 718, 1001

Bournaud, F., Valentin Perret, Renaud, F., et al. 2014, Astrophys. J., 780, 57

Chartas, G., Hamann, F., Eracleous, M., et al. 2014, Astrophys. J., 783, 57

Dubois, Y., \& Teyssier, R. 2008, ASP Conf. Ser., 390, 388

Gabor, J. M., \& Bournaud, F. 2013, Mon. Not. R. Astron. Soc., 16, 1

-. 2014, Mon. Not. R. Astron. Soc., 13, 1

Hopkins, P. F., Kereš, D., Oñorbe, J., et al. 2014, Mon. Not. R. Astron. Soc., 445, 581

Renaud, F., Bournaud, F., Emsellem, E., et al. 2013, Mon. Not. R. Astron. Soc., 436, 1836

Roos, O., Juneau, S., Bournaud, F., \& Gabor, J. M. 2015, Astrophys. J., 800, 19

Sommer-Larsen, J. 2006, Astrophys. J., 644, L1

Steidel, C. C., Erb, D. K., Shapley, A. E., et al. 2010, Astrophys. J., 717, 289 